Supporting Information

\title{
Effect of Additives on Hydrothermal Liquefaction of Polysaccharides
}

\author{
Akhila Gollakota and Phillip E. Savage* \\ Department of Chemical Engineering \\ 121D Chemical and Biomedical Engineering Building
}

The Pennsylvania State University,

University Park, PA 16802, United States

* psavage@psu.edu 
Table S1 CHNO content (wt\%) and higher heating value (HHV) for polysaccharides and HTL biocrudes without additives. Data are from Table 2 in Ref 2.

\begin{tabular}{|c|c|c|c|c|c|c|c|c|}
\hline & & & $\mathrm{C}$ & $\mathrm{H}$ & $\mathrm{N}^{\ddagger}$ & $\mathrm{O}^{*}$ & $\begin{array}{l}\mathrm{HHV} \\
(\mathrm{MJ} / \mathrm{kg})\end{array}$ & $\begin{array}{l}\text { Energy } \\
\text { recovery }\end{array}$ \\
\hline & & \multicolumn{7}{|c|}{ Polysaccharides } \\
\hline \multicolumn{3}{|l|}{ Starch } & 37.0 & 7.7 & 0.1 & 55.2 & 17.0 & - \\
\hline \multicolumn{3}{|l|}{ Amylopectin } & 39.4 & 8.0 & 0.1 & 52.5 & 18.6 & - \\
\hline \multicolumn{3}{|l|}{ Amylose } & 40.5 & 8.2 & 0.2 & 51.1 & 19.3 & - \\
\hline \multicolumn{3}{|l|}{ Cellulose } & 42.3 & 7.6 & 0.1 & 50.0 & 19.3 & - \\
\hline \multicolumn{3}{|l|}{ Pectin } & 38.9 & 7.0 & 0.2 & 53.9 & 17.6 & - \\
\hline \multicolumn{3}{|l|}{ Chitin } & 44.5 & 6.6 & 6.6 & 42.3 & 18.7 & - \\
\hline Feedstock & $\begin{array}{l}\text { Set-point } \\
\mathrm{T}\left({ }^{\circ} \mathrm{C}\right)\end{array}$ & $\begin{array}{l}\text { Time } \\
\text { (min) }\end{array}$ & \multicolumn{6}{|c|}{ Biocrudes } \\
\hline \multirow[t]{3}{*}{ Starch } & 350 & 5.6 & 62.2 & 7.4 & 0.1 & 30.4 & 28.2 & 26.9 \\
\hline & 350 & 31.6 & 64.9 & 8.0 & 0.1 & 27.1 & 30.4 & 36.4 \\
\hline & 500 & 1 & 62.8 & 8.3 & 0.1 & 28.7 & 30.0 & 41.1 \\
\hline \multirow[t]{3}{*}{ Amylopectin } & 350 & 5.6 & 62.6 & 7.1 & 0.1 & 30.2 & 27.9 & 20.0 \\
\hline & 350 & 31.6 & 71.0 & 7.5 & 0.1 & 21.5 & 32.4 & 30.8 \\
\hline & 500 & 1 & 65.6 & 8.2 & 0.0 & 26.2 & 31.0 & 38.1 \\
\hline \multirow[t]{3}{*}{ Amylose } & 350 & 5.6 & 55.6 & 7.4 & 0.1 & 36.9 & 25.3 & 17.1 \\
\hline & 350 & 31.6 & 70.9 & 7.8 & 0.1 & 21.2 & 32.9 & 31.8 \\
\hline & 500 & 1 & 64.7 & 7.1 & 0.1 & 28.1 & 28.9 & 32.7 \\
\hline \multirow[t]{3}{*}{ Cellulose } & 350 & 5.6 & 62.0 & 6.8 & 0.1 & 31.1 & 27.2 & 20.2 \\
\hline & 350 & 31.6 & 69.8 & 8.6 & 0.1 & 21.4 & 33.8 & 32.4 \\
\hline & 500 & 1 & 62.7 & 7.8 & 0.1 & 29.4 & 29.1 & 33.6 \\
\hline \multirow[t]{3}{*}{ Pectin } & 350 & 5.6 & 63.2 & 7.7 & 0.1 & 29.0 & 29.2 & 21.7 \\
\hline & 350 & 31.6 & 66.8 & 8.6 & 0.1 & 24.6 & 32.2 & 25.4 \\
\hline & 500 & 1 & 63.8 & 7.2 & 0.1 & 28.9 & 28.7 & 29.5 \\
\hline \multirow[t]{3}{*}{ Chitin } & 350 & 5.6 & 67.9 & 7.2 & 5.9 & 19.0 & 30.6 & 25.1 \\
\hline & 350 & 31.6 & 74.5 & 8.3 & 5.2 & 12.0 & 35.4 & 37.0 \\
\hline & 500 & 1 & 73.3 & 8.5 & 6.9 & 11.4 & 35.1 & 45.2 \\
\hline
\end{tabular}

$\$$ only chitin contains $\mathrm{N}$. The non-zero values for the other polysaccharides and their HTL biocrudes are within the experimental accuracy and are taken as effectively zero.

*calculated by difference, data for chitin are from Ref 13. 
Table S2 Elemental composition (wt \%) and HHVs of biocrudes from isothermal and fast HTL of polysaccharides with additives. $5 \mathrm{wt} \%$ biomass loading, 10:1 mass ratio of biomass to additive

\begin{tabular}{|c|c|c|c|c|c|c|c|c|}
\hline Additive & Feedstock & $\begin{array}{l}\text { Set-point } \\
\mathrm{T}\left({ }^{\circ} \mathrm{C}\right)\end{array}$ & $\begin{array}{l}\text { Time } \\
(\min )\end{array}$ & $\mathrm{C}$ & $\mathrm{H}$ & $\mathrm{N}$ & $\mathrm{O}^{*}$ & $\begin{array}{c}\mathrm{HHV} \\
(\mathrm{MJ} / \mathrm{kg})\end{array}$ \\
\hline \multirow[t]{18}{*}{$\mathrm{K}_{2} \mathrm{CO}_{3}$} & \multirow[t]{3}{*}{ Cellulose } & \multirow[t]{2}{*}{350} & 5.6 & 71.3 & 7.9 & 0.1 & 20.6 & 33.3 \\
\hline & & & 31.6 & 74.3 & 8.2 & 0.1 & 17.4 & 35.2 \\
\hline & & 500 & 1 & 70.7 & 7.9 & 0.1 & 21.3 & 33.1 \\
\hline & \multirow[t]{3}{*}{ Starch } & \multirow[t]{2}{*}{350} & 5.6 & 66.1 & 7.2 & 0.2 & 26.5 & 29.8 \\
\hline & & & 31.6 & 73.8 & 8.3 & 0.1 & 17.8 & 35.1 \\
\hline & & 500 & 1 & 67 & 7.5 & 0.1 & 25.3 & 30.7 \\
\hline & \multirow[t]{3}{*}{ Amylose } & \multirow[t]{2}{*}{350} & 5.6 & 70.5 & 7.7 & 0.1 & 21.7 & 32.6 \\
\hline & & & 31.6 & 78 & 8.6 & 0.1 & 13.3 & 37.5 \\
\hline & & 500 & 1 & 75 & 8.6 & 0.1 & 16.4 & 36 \\
\hline & \multirow[t]{3}{*}{ Amylopectin } & \multirow[t]{2}{*}{350} & 5.6 & 68.3 & 8.4 & 0.1 & 23.2 & 32.6 \\
\hline & & & 31.6 & 72.8 & 7.2 & 0.3 & 19.7 & 32.9 \\
\hline & & 500 & 1 & 67.6 & 7.8 & 0.2 & 24.4 & 31.3 \\
\hline & \multirow[t]{3}{*}{ Chitin } & \multirow[t]{2}{*}{350} & 5.6 & 53.1 & 8.9 & 4.7 & 33.3 & 26.3 \\
\hline & & & 31.6 & 58.4 & 8.2 & 5.5 & 27.8 & 27.8 \\
\hline & & 500 & 1 & 54.1 & 9.6 & 4.8 & 31.5 & 27.9 \\
\hline & \multirow[t]{3}{*}{ Pectin } & \multirow[t]{2}{*}{350} & 5.6 & 55.6 & 6.2 & 0.5 & 37.8 & 23.3 \\
\hline & & & 31.6 & 55.2 & 5.9 & 0.5 & 38.3 & 22.7 \\
\hline & & 500 & 1 & 54.3 & 6.1 & 0.4 & 39.3 & 22.4 \\
\hline \multirow[t]{18}{*}{$\mathrm{HCOOH}$} & \multirow[t]{3}{*}{ Cellulose } & \multirow[t]{2}{*}{350} & 5.6 & 71.1 & 6.9 & 0.1 & 21.8 & 31.7 \\
\hline & & & 31.6 & 79.5 & 8.4 & 0.1 & 12 & 37.8 \\
\hline & & 500 & 1 & 53.9 & 5.4 & 0.1 & 40.6 & 21.3 \\
\hline & \multirow[t]{3}{*}{ Starch } & \multirow[t]{2}{*}{350} & 5.6 & 68.1 & 7 & 0.1 & 24.7 & 30.4 \\
\hline & & & 31.6 & 74.2 & 7.8 & 0.1 & 17.9 & 34.4 \\
\hline & & 500 & 1 & 59 & 6.3 & 0.1 & 34.6 & 25 \\
\hline & \multirow[t]{3}{*}{ Amylose } & \multirow[t]{2}{*}{350} & 5.6 & 67.9 & 6.5 & 0.1 & 25.4 & 29.6 \\
\hline & & & 31.6 & 73.9 & 10.8 & 0.1 & 15.2 & 39 \\
\hline & & 500 & 1 & 56.6 & 5.9 & 0.3 & 37.2 & 23.4 \\
\hline & \multirow[t]{3}{*}{ Amylopectin } & \multirow[t]{2}{*}{350} & 5.6 & 76.4 & 8 & 0.1 & 15.4 & 35.9 \\
\hline & & & 31.6 & 79.3 & 9.1 & 0.3 & 11.3 & 38.9 \\
\hline & & 500 & 1 & 53.3 & 5.7 & 0.1 & 40.8 & 21.5 \\
\hline & \multirow[t]{3}{*}{ Chitin } & \multirow[t]{2}{*}{350} & 5.6 & 68.6 & 8.3 & 7.1 & 16.1 & 32.6 \\
\hline & & & 31.6 & 71.6 & 9.2 & 6.2 & 13 & 35.4 \\
\hline & & 500 & 1 & 62.7 & 6.9 & 5.5 & 24.8 & 27.8 \\
\hline & \multirow[t]{3}{*}{ Pectin } & \multirow[t]{2}{*}{350} & 5.6 & 72.1 & 7.8 & 0.6 & 19.4 & 33.6 \\
\hline & & & 31.6 & 79.8 & 9.3 & 0.6 & 10.3 & 39.4 \\
\hline & & 500 & 1 & 59 & 6.3 & 0.1 & 34.6 & 25 \\
\hline
\end{tabular}

$\$$ only chitin contains N. The non-zero values for the biocrudes from other polysaccharides are typically within the experimental accuracy and are taken as effectively zero

*calculated by difference. 
Table S3 Distribution of C (\%) into biocrude and aqueous phase after HTL without and with additives

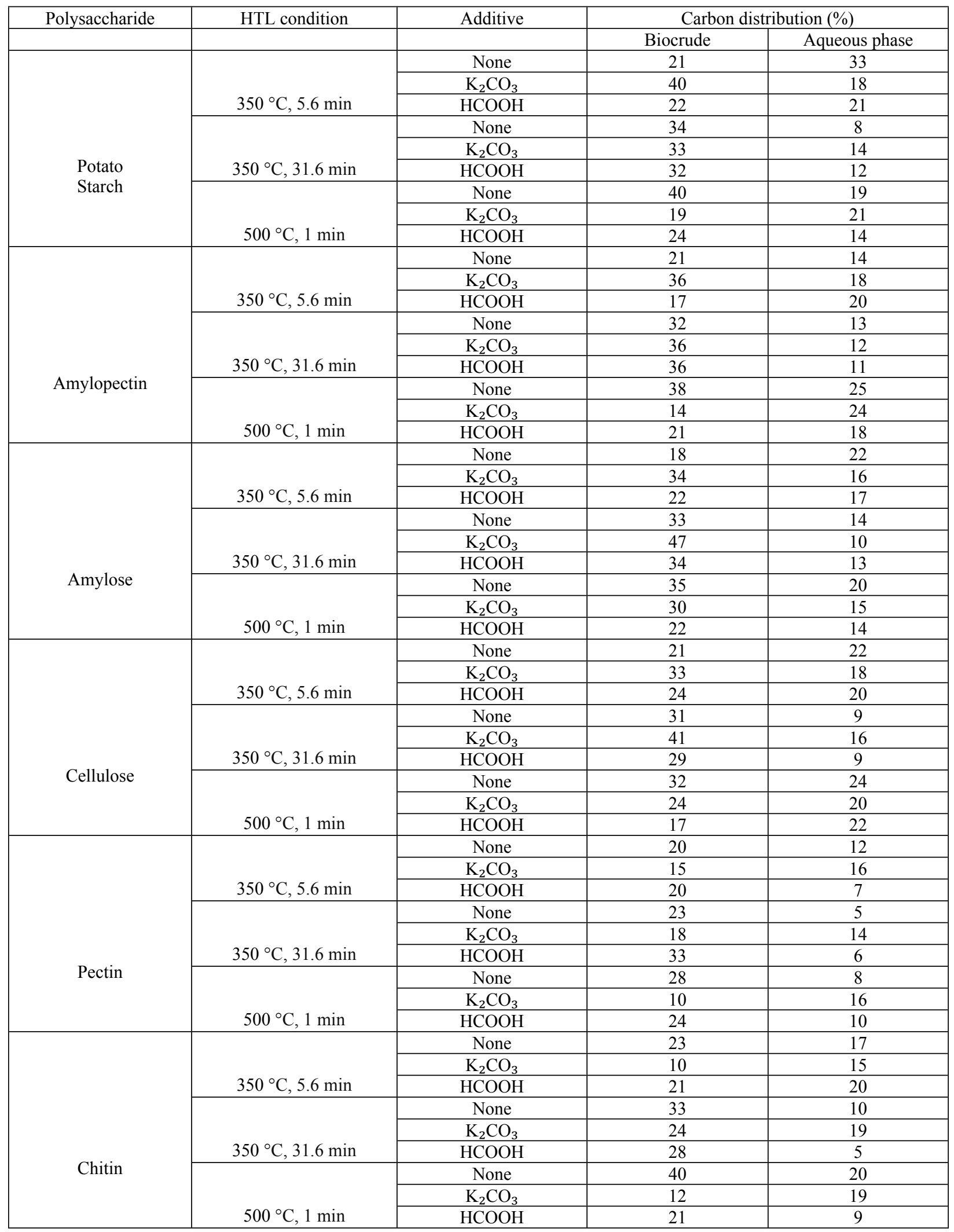

